PostScript

LETTERS

\section{Antenatal maternal transfers in the east of England: role of a centralised cot bureau}

There are anecdotal reports of expecting mothers, likely to deliver an infant requiring neonatal intensive care, transferred between hospitals because of lack of local neonatal intensive care capacity. However, there are no data on the scale of this practice. The job of locating an admitting hospital often befalls the clinical staff on busy delivery units, taking them away from direct clinical care. Although this activity does not involve neonatal staff directly, it does raise questions about appropriate management of neonatal intensive care unit (NICU) resources locally especially with the development of neonatal networks. Recognising these issues, the regional neonatal transport service extended the role of the neonatal cot bureau to assist regional delivery units in locating suitable admitting hospitals. Starting in November 2003, and working from 0800 to 2030, the bureau operators asked the referring clinicians standard questions, including reasons for the transfer, the gestational age, as well as complications of pregnancy. The operators then phoned hospitals within and, if necessary, outside of the eastern region to locate one with suitable facilities. Once an admitting unit was identified, its contact details were relayed to the referring clinician for further discussions and transfer to take place. The bureau phoned the referring unit later to confirm if the transfer took place. We would like to share the results of this activity over the calendar year 2004

All 18 hospitals in the region used the service, making 262 transfer requests. Admitting units were identified in $179(68 \%)$ cases; $42 \%$ of these units were outside of the region. A mean (range) of 7.6 (1-36) hospitals were contacted before an admitting unit could be located. Mean (range) time to locate the unit was 45 (6-215) minutes. Reasons for transfer request included: both delivery and NICU full locally, $11 \%$; delivery unit full, $1.5 \%$; NICU full, $54 \%$; baby expected to be too premature for local NICU, $26 \%$; other, $7 \%$. Mean (range) gestational age at the time of transfer request was $29.5(23-40)$ weeks. A total of 165 women were successfully transferred. These patients were transferred over a mean (range) distance of 66.5 (12-250) miles with mean (range) estimated journey time of 80 (14-300) minutes. Of the 85 women not transferred, no suitable admitting units could be located for $43 \%$, transfer request was withdrawn by the referring unit in $38 \%, 11 \%$ delivered before they could be transferred, and admitting units declined after initial acceptance in $2 \%$. No reason for non-transfer could be ascertained by the bureau in $5 \%$ of cases.

This service is the only one of its kind in the United Kingdom. Although it does not capture the entire regional antenatal transfer activity, it has been of assistance to clinicians in the front line. These data suggest that maternal transfers take place regularly, often over long distances. Finding a suitable admitting unit is a time consuming task. Lack of local neonatal capacity and expertise is a major underlying cause. While we await the results of current reconfiguration of maternal and neonatal care provision in the country, services similar to ours can help better management of available clinical resources.
I U Cheema, R K Bomont

Correspondence to: Dr Cheema, Acute Neonatal Transport Service, Box 224, Addenbrookes Hospital NHS Foundation Trust, Hills Road Cambridge CB2 2QQ, UK; Irfan.cheema@addenbrookes.nhs.uk

\section{doi: 10.1136/adc. 2005.076448}

\section{Yes, human milk does reduce infection rates in very low birthweight infants}

Drs De Silva, Jones, and Spencer have provided a timely review of issues relating to studies of breast feeding for very low birthweight (VLBW) infants and rates of infection. ${ }^{1}$ We would appreciate the opportunity to clarify some points that they have raised with regard to our study. ${ }^{2}$ We believe our results clearly support our conclusion: that intake of more than $50 \mathrm{ml} / \mathrm{kg} /$ day maternal milk through week 4 of life decreases the rate of sepsis in VLBW infants.

de Silva et al state that, “... a major flaw noted in all the studies was the lack of a consistent definition of HM fed groups or methods used to quantify HM intake." We were able to provide a concise definition of maternal milk intake, which was classified by mean $\mathrm{ml} / \mathrm{kg} /$ day through week 4 of life, and included groups fed $1-24 \mathrm{ml} / \mathrm{kg} /$ day, $\quad 25-49 \mathrm{ml} / \mathrm{kg} /$ day, and $\geqslant 50 \mathrm{ml} / \mathrm{kg} /$ day. These groups were compared with a reference group receiving $0 \mathrm{ml} / \mathrm{kg} /$ day maternal milk. We also describe the maternal milk intake of our population in two week increments by daily volume, by total volume, as a percentage of total intake, and as a percentage of enteral intake. We agree that, when human milk intake is measured only as a proportion of enteral intake, infants may be misclassified, and state this in our discussion.

de Silva et al criticise our work and that of others for not including an exclusively human milk fed cohort. The realities of breast feeding for VLBW infants, including months of milk expression, make attaining a cohort of exclusively human milk fed infants extremely difficult. As de Silva et al point out, not having such a cohort is likely to lead to an underestimate of the beneficial effects of human milk, and strengthens rather than weakens our (and others') conclusion that human milk reduces infection rates.

We agree that including a wide range of infections other than sepsis, such as urinary tract infection and pneumonia, may confuse rather than clarify an effect of maternal milk. We examined solely the rate of sepsis, and defined it as "a positive blood culture obtained in the presence of clinical signs and/or symptoms of infection, treated for five or more days with antibiotics". This is the gold standard. As inflammatory response to infection in VLBW infants may be lacking, may lag, and is not always an accurate measure of illness severity, the addition of data on inflammatory markers and changes in cell counts would not be expected to provide clarification.

We also agree with de Silva et al that the timing of maternal milk intake in relation to onset of infection is important, and we examined rates of sepsis after weeks 2, 4, and 6 of life according to cumulative volume of maternal milk received before these ages. In a logistic regression that adjusted for birth weight, we found that "the greater the average volume of maternal milk intake $(\mathrm{ml} / \mathrm{kg} /$ day $)$, the lower the rate of sepsis after weeks two and four (both $\mathrm{p}<.05$ ), though not after week six." We also excluded (throughout the study) all episodes of sepsis occurring before day 5 of life, as enteral intake was minimal before this time.

De Silva et al are correct that postnatal steroid intake may affect the rate of infection, and we did not control for this in our study. As $27 \%$ of our population received postnatal steroid treatment, which was standard of care at the time, this is an important consideration and a valid criticism. We have performed again the Poisson regression analysis that examines the effect of maternal milk on the rate of sepsis. While also controlling for birth weight, race, and sex as in the original analysis, we controlled for postnatal steroid treatment as well. This resulted in negligible changes in the relative risk and confidence intervals for each (maternal milk intake) group. The effect of postnatal steroid treatment in this analysis was not significant, and our original conclusions are unchanged.

Finally, there is no question that future research should include larger numbers of infants. When our project was submitted for funding, we calculated our sample size based on rate of sepsis as the primary outcome measure of interest. Specifically our proposal read: "Based on the sample size of 60 infants in each group, we will have the ability to detect a $50 \%$ decrease in the rate of sepsis, i.e. from $40 \%$ to $20 \%$ (power $=.78$, alpha one tailed $=.05)^{\prime \prime}$. de Silva et al state that "...64 [infants] are required in each group with an addition for drop out..." Their and our calculations are quite similar. The difficulty we encountered is that, although more than half of mothers intended to breast feed (87 mothers, 73\%), 18 had discontinued lactation by 3 weeks postnatal age, and 39 more by 40 weeks corrected age. ${ }^{3}$ Other centres experienced similar rates of discontinuation during the same time period. ${ }^{45}$ Not only is a randomised trial of breast feeding unethical, it remains a challenge simply to support those mothers who have enrolled and chosen to breast feed. Further work is needed.

We believe that our study clearly shows that VLBW infants who receive more than $50 \mathrm{ml} /$ $\mathrm{kg}$ /day maternal milk through the first 4 weeks of life have a decreased rate of sepsis, and thus maternal milk feeding is beneficial for these infants.

L Furman

Department of Pediatrics, Case Western Reserve University School of Medicine, Rainbow Babies and Children's Hospital, 11100 Euclid Avenue, Mather 3 Cleveland OH 44106, USA; Imfurman@adelphia.net

\section{References}

1 de Silva A, Jones PW, Spencer SA. Does human milk reduce infection rates in preterm infants? A systematic review. Arch Dis Child Fetal Neonatal Ed 2004;89:F509-13.

2 Furman L, Taylor G, Minich N, et al. The effect of maternal milk on neonatal morbidity of very low birth weight infants. Arch Pediatr Adolesc Med 2003;157:66-71.

3 Furman L, Minich N, Hack M. Correlates of lactation in mothers of VLBW infants. Pediatr 2002;109.www. pediatrics.org/cgi/content/full/ 109/4/e57.

4 Bier JB, Ferguson AE, Morales $Y$, et al Breastfeeding infants who were extremely low birthweight. Pediatrics, 1997;100 www.pediatrics.org/cgi/content/full/100/61/e3.

5 Richards MT, Lang MD, Mclntosh C, et al. Breastfeeding the VLBW infant: successful outcome and maternal expectations [abstract] Pediatr Res 1996;39:383A. 